\title{
Cómo el sexo moldea la evolución del comportamiento: el caso de las preferencias en la reproducción humana
}

\author{
Leonardo Moreno Naranjo * y Germán Gutiérrez
}

Departamento de Psicología, Universidad Nacional de Colombia, Bogotá, Colombia

Recibido el 2 de marzo de 2020; aceptado el 2 de noviembre de 2020

\section{PALABRAS CLAVE Preferencias, selección sexual, selección de pareja}

\begin{abstract}
Resumen La reproducción sexual es uno de los grandes hitos de la evolución de la vida en la Tierra; si bien pudo facilitar la generación de variabilidad genética, también impuso una serie de retos adaptativos en los organismos, tales como la búsqueda de parejas y las estrategias para asegurar la reproducción. La teoría de selección sexual ofrece hipótesis que permiten explicar cómo la búsqueda y la selección de parejas no son aleatorias o arbitrarias en los organismos complejos. Se plantea que existen unos criterios para seleccionar parejas reproductivas que se ven reflejados en mecanismos psicológicos denominados preferencias, las cuales pueden orientar la elección de parejas que tengan rasgos adaptativos y beneficiar o bien la supervivencia, o bien la reproducción futura de la descendencia que herede tales caracteres. El presente artículo aborda cómo las condiciones que impone la sexualidad moldean la evolución del comportamiento y cómo aquellos principios se aplican al emparejamiento humano. A partir de esto se plantean las principales preguntas que derivan de esta relación entre evolución, preferencias y selección de pareja.

(c) 2021 Fundación Universitaria Konrad Lorenz. Este es un artículo Open Access bajo la licencia CC BY-NC-ND (http://creativecommons.org/licenses/by-nc-nd/4.0/).
\end{abstract}

How sex shapes the evolution of behavior: The case of preferences in human reproduction

Abstract Sexual reproduction is one of the greatest landmarks of the evolution of life on Earth. Although it helped the generation of genetic variability, it also imposed a series of adaptive challenges in organisms, such as the search for mates and strategies to secure reproduction. The theory of sexual selection offers hypotheses to explain that the search and selection of a mate are not arbitrary for complex organisms. It is proposed that there are criteria for selecting reproductive mates that are reflected in psychological mechanisms called preferences, which can guide the choice of partners who have adaptive traits and can benefit, either survival or future reproduction of the offspring that inherit such traits. This review addresses how the conditions imposed by sexuality shape the evolution of behavior, and how those principles apply to human mating behavior. From there, important questions arise to understand the relationship among evolution, preferences and mate selection.

(c) 2021 Fundación Universitaria Konrad Lorenz. This is an open access article under the CC BY-NCND license (http://creativecommons.org/licenses/by-nc-nd/4.0/).

\footnotetext{
* Autor para correspondencia.

Correo electrónico: Imorenon@unal.edu.co
} 
Se estima que alrededor de un millón trescientas mil especies se reproducen sexualmente; sin embargo, trazar una línea definida respecto a la historia evolutiva de esta forma de reproducción se ha convertido en uno de los más grandes retos para los teóricos de la evolución. La reproducción sexual ofrece aparentes ventajas a la hora de promover variabilidad genética en la descendencia, y esta variabilidad es la materia prima de la evolución de las especies (i.e., selección natural); no obstante, esta forma de reproducción tiene algunos costos, tales como la producción de gametos sexuales, un gasto de energía en la búsqueda y selección de una pareja reproductiva, el esfuerzo cooperativo que demanda y el potencial conflicto intrasexual e intersexual que deriva de ella (Andersson, 1994).

Dado que es un proceso costoso para los individuos, ¿por qué prevalece la reproducción sexual en la historia de vida de las especies? Responder a esta pregunta requiere analizar qué condiciones ecológicas pudieron favorecer la aparición y el mantenimiento de este sistema a lo largo del tiempo y de qué manera se pudo extender a otras especies "complejas" actualmente vivas. Sin embargo, la información que se tiene sobre el posible origen de la reproducción sexual es limitada, en razón a la dificultad que representa estudiar los posibles vestigios de los primeros organismos sexuales y examinar a aquellos organismos simples actuales que presentan rudimentariamente este sistema reproductivo. Algunas hipótesis clásicas (Maynard-Smith, 1978) sugieren varios beneficios que pudieron ser críticos en la permanencia de los mecanismos sexuales; por ejemplo, en situaciones en las que las condiciones ambientales cambian de forma constante, las poblaciones genéticamente diversas que se reproducen sexualmente cuentan con ventaja para adaptarse a las nuevas presiones ecológicas; así, la reproducción asexual ofrecería poblaciones menos diversas y, en consecuencia, una más baja adaptación a cambios ambientales bruscos o frecuentes (Schurko et al., 2009).

Aun así, investigaciones recientes basadas en simulaciones y modelos matemáticos sugieren que la reproducción sexual no necesariamente promueve una mayor variabilidad genética con respecto a la reproducción asexual y, a pesar de ser predominante en organismos complejos, puede ser opcional (Hadany \& Otto, 2009; Zimmer, 2009). Por ejemplo, crustáceos del género Dhapnia se reproducen asexualmente cuando los recursos son abundantes y cambian a la reproducción sexual cuando hay parejas disponibles y las condiciones son más adversas, así como cuando hay patógenos en el ambiente (Kleiven et al., 1992).

De acuerdo con los problemas que se derivan del argumento anterior se plantea una nueva pregunta: ¿por qué la reproducción sexual es predominante en especies complejas? Una posible respuesta es que los organismos sexuales se reprodujeron a tasas más altas que los asexuales, debido a que estos seres explotaban algunas características llamativas que los hacían atractivos y tuvieron la posibilidad de acaparar un número creciente de parejas, lo cual representó que los genes de los individuos con reproducción sexual se propagaran más rápido en las siguientes generaciones, tal como parece demostrarse en simulaciones con especies con reproducción facultativa (Hadany \& Beker, 2007; Hadany \& Otto, 2009).

Además de la variabilidad genética, otros investigadores sugieren que la reproducción sexual también es un mecanismo reparador de los genes (Bernstein et al., 2012). Dado que el proceso de replicación de los genes no es perfecto $y$, en ocasiones, se presentan fallos en la duplicación de las secuencias genéticas o de compatibilidad en los cromosomas, el proceso de recombinación genética presente en la reproducción sexual puede enmascarar las mutaciones perjudiciales y prevenir que se manifiesten en las siguientes generaciones. En contraste, en el caso de la reproducción asexual, los hijos, al ser genéticamente semejantes a sus padres, heredarían los genes defectuosos; al no contar con mecanismos reproductivos como la meiosis, las mutaciones perjudiciales se irían acumulando a través de las generaciones, si bien las presiones evolutivas terminarían por reducir la cantidad de individuos con estas mutaciones en la población. En resumen, la reproducción sexual ayudaría a reducir la carga de mutaciones perjudiciales con el paso de las generaciones, mientras que en la reproducción asexual estas mutaciones se acumularían (Maynard-Smith, 1978).

Aparte de adaptaciones anatomo-fisiológicas, la reproducción sexual implica adaptaciones comportamentales, de modo que se integran como una parte fundamental del ciclo vital de los organismos y contribuyen así a definir la dirección misma de la evolución por medio de la delimitación de los criterios con los que se elige una pareja reproductiva.

Gracias a los procesos evolutivos se han fijado algunos mecanismos para la selección de parejas que delimitan patrones específicos de selección y ayudan a definir qué es lo atractivo y qué no lo es en una potencial pareja; a estos mecanismos se les conoce como preferencias. Asimismo, las preferencias cumplen también una función motivacional, ya que incitan a los individuos a buscar parejas con ciertas características, cuya percepción determina como atractivas. En general, esas características "atractivas" pueden suponer un beneficio adaptativo, aunque pueden surgir características que exploten las cualidades perceptivas de los individuos y atraigan también parejas reproductivas, más allá de los beneficios mencionados (Ryan, 2018). Estos problemas los tratan las teorías de selección sexual e inversión parental.

\section{Selección sexual y evolución del comportamiento}

La selección sexual ha sido descrita como un mecanismo derivado de la selección natural en el que se promueve el cambio evolutivo por medio de un factor selector asociado a una característica reproductiva; en otras palabras, en la selección sexual el criterio de selección no son las exigencias ambientales, sino características de los individuos que representen una ventaja para mejorar la probabilidad del éxito reproductivo (Gutiérrez, 2010; Matthews \& Domjan, 2011). Este mecanismo fue descrito por Darwin (1871) a partir de la observación de algunas características en animales que aparentemente suponían una desventaja para la supervivencia de los individuos, pero podrían tener una función relacionada con la reproducción; al ser vistosos, pensó Darwin, posiblemente ayudarían a atraer parejas sexuales. En el clásico ejemplo de la larga y vistosa cola de los pavos reales (Pavo cristatus) puede haber un costo al convertir a los machos en un blanco fácil para los depredadores; no obstante, también los convierte en altamente deseables para las hembras y asegurar la reproducción.

La teoría de selección sexual describe dos componentes: selección intrasexual y selección intersexual. En la selección 
intrasexual los individuos de un mismo sexo compiten entre sí por la atención y el acceso reproductivo de parejas del sexo opuesto. Tal competencia se puede dar en forma de una mayor capacidad para identificar o acceder a parejas (Gutiérrez \& Domjan, 1996), encuentros agresivos entre machos, despliegues de cortejo, demostración de habilidades que logren la atención de potenciales parejas, exhibición de rasgos llamativos $y$, en especies sociales, el rango o estatus social de los individuos en la jerarquía (Andersson, 1994). También puede expresarse en el proceso copulatorio, mediante un grupo de fenómenos que en su conjunto se denominan competencia espermática y deriva de la situación potencial en la que una hembra se aparee con más de un macho durante el periodo reproductivo. Adaptaciones anatómicas, fisiológicas y conductuales pueden facilitar la capacidad de fecundación por parte de un macho en un contexto de competencia espermática entre dos o más machos en especies diversas (Gutiérrez \& Ortiz, 2002; Simmons \& Fitzpatrick, 2012). También puede expresarse como cuidado de la pareja durante el periodo reproductivo, especialmente en el periodo poscopulatorio (Shackelford et al., 2006).

El segundo componente de la selección sexual se denomina selección intersexual e involucra la evaluación y la selección de parejas por medio de las preferencias. Los individuos evalúan a sus parejas a partir de unos criterios de selección ligados a características o habilidades en las que compiten los individuos del sexo opuesto (Trivers, 1972). Al seleccionar con quién reproducirse están definiendo características que contribuyan a la reproducción o los rasgos que serán heredados por las crías.

Tanto la selección intersexual como la competencia intrasexual pueden interactuar al determinar qué preferencias de los individuos (usualmente de las hembras) definen los dominios en los que deben competir los miembros del sexo opuesto y, al mismo tiempo, la competencia entre individuos fija las características que pueden ser deseables para sus compañeros sexuales; este proceso se conoce como runaway selection o "selección en cascada" (Fisher, 1930) y permite analizar cómo las características sexuales secundarias y las preferencias por estas pudieron desarrollarse en la historia evolutiva de las especies. Se parte de la idea de que los individuos enfrentan el problema de escoger a la mejor pareja del sexo opuesto y que algunos rasgos serán deseables debido a que ofrecen una demostración de las cualidades biológicas de los sujetos que, adicionalmente, pueden ser heredables.

La marcada preferencia por algunos rasgos incrementa su frecuencia en las siguientes generaciones e incluso promueve variantes cada vez más exageradas de los rasgos atractivos, potencialmente incrementando el costo de mantener esos atributos y comprometiendo la supervivencia del individuo. Al final, la selección natural se encarga de balancear la expresión de estas cualidades, en la medida en que los individuos con caracteres demasiado costosos no sobreviven para reproducirse.

Hasta este punto se han mencionado de forma indirecta patrones asociados con las diferencias sexuales; por ejemplo, usualmente los machos compiten por las hembras y las hembras son las que eligen con quien reproducirse. Hacia mediados del siglo XX, los biólogos evolutivos trataron de buscar explicaciones a este problema y las posibles condiciones que lo facilitan. Robert Trivers (1972) propuso entonces la teoría de inversión parental, la cual complementa las hipótesis de Darwin y extiende el horizonte investigativo de la reproducción.

\section{El papel de la inversión parental}

La teoría de inversión parental propone un modelo de explicación que integra las nociones con respecto al uso óptimo de recursos para asegurar que los genes de cada individuo se vean representados en posteriores generaciones; este objetivo ha sido denominado eficacia biológica o fitness (Hamilton, 1964). Al plantear que cada organismo tiene la tendencia a mejorar su eficacia biológica, es lógico pensar que los individuos tratarán de maximizar su capacidad reproductiva, e incluso mejorar el éxito reproductivo de la descendencia.

Existen diferencias entre los sexos en la forma de proveer recursos reproductivos con el fin de maximizar la eficacia biológica individual; esa inversión de recursos no solo se expresa en el cortejo y la copula, sino también en la crianza de los hijos (Trivers, 1972). El punto central es que machos y hembras cooperan en la reproducción, pero también buscan reducir los costos de inversión en sus crías a costa del esfuerzo de la pareja, con el fin de tener otras oportunidades de reproducción y mejorar su eficacia biológica. Así, por ejemplo, en los mamíferos las hembras hacen una mayor inversión parental que se manifiesta en un tiempo de gestación prolongado y una inversión energética en la protección y alimentación de las crías (Carrillo, 2011; Daly \& Perry, 2018).

A partir de lo anterior, se plantea que el sexo que más invierte en las crías tiende a ser más selectivo, debido a que necesita compensar los costos de su inversión a priori escogiendo miembros del sexo opuesto que tengan aptitudes sobresalientes e interés en invertir recursos en las parejas o en las crías; por otra parte, el sexo que menos invierte en las crías suele presentar una mayor competencia intrasexual, además de tener una mayor inclinación por acceder a una gran cantidad de parejas sexuales, con el fin de maximizar su eficacia biológica.

Estas diferencias en inversión parental también se ven reflejadas en las preferencias o criterios de selección de pareja. Por ejemplo, en el caso de numerosos mamíferos, las hembras son atraídas por aquellos machos que tienen la capacidad de proveer recursos para ellas y sus crías, y que ofrezcan protección o un alto estatus, en el caso de las especies sociales. Por otra parte, los machos mamíferos son atraídos por hembras que muestran indicadores de fertilidad y salud. Por supuesto, las preferencias mencionadas no son exclusivas de cada sexo, pero se ven enfatizadas dependiendo de su grado de inversión parental; en aquellas especies que presentan una inversión parental equivalente, las preferencias tienden a ser similares en ambos sexos (Trivers, 1972).

La inversión parental también determina las estrategias y los sistemas de apareamiento en especies animales. Las especies que tienden a emparejarse "a largo plazo" presentan patrones tales como la monogamia y la poligamia. En las relaciones a largo plazo se enfatiza el papel de los progenitores en la provisión de recursos y la protección tanto para la pareja como para las crías por largos periodos de tiempo. En estos casos, los individuos suelen ser más selectivos con sus parejas, ya que una mala decisión podría perjudicar su eficacia biológica personal. 
En algunos tipos de emparejamiento la inversión parental se reduce en uno de los dos sexos o en ambos. En las estrategias de emparejamiento "a corto plazo" como la promiscuidad, la monogamia serial o los patrones de infidelidad, se establecen relaciones sin exclusividad para el acceso sexual, teniendo en cuenta que se buscan encuentros breves y con bajo compromiso. En esta situación se da preferencia tanto al acceso inmediato de recursos como a la calidad genética de los individuos; estos criterios ayudan a que se maximice la inversión en forma de cualidades sobresalientes que pueden heredar los hijos. Las estrategias a corto plazo se presentan con más frecuencia en ambientes inestables, en los cuales la obtención de recursos y el acceso a las parejas son impredecibles (Pianka, 1970).

\section{Retos reproductivos en los humanos y sus consecuencias en el comportamiento}

En el caso humano, varios de los elementos mencionados se presentan en forma de problemas reproductivos, comunes a otras especies, pero que los humanos abordan con una alta flexibilidad, como lo es el caso de la adaptación a los diversos patrones de emparejamiento presentes en la naturaleza, mediante lo que algunos teóricos consideran un patrón mixto de emparejamiento (Gangestad \& Simpson, 2000).

La teoría de estrategias sexuales, desarrollada por Buss y Schmitt (1993), plantea que durante la historia evolutiva de los humanos han aparecido una serie de adaptaciones para resolver distintos problemas reproductivos dependiendo del sexo o el sistema de apareamiento por el cual optan los individuos. Las estrategias orientadas a resolver aquellos problemas son muy variadas; incluyen mecanismos psicológicos para la evitación de infidelidades dirigidas a enfrentar el problema de certeza de la paternidad (Buss, 2018; Buss \& Shackelford, 1997), las cuales pueden abarcar adaptaciones más generales como las que se basan en la preferencia de parejas que demuestren rasgos de lealtad (Lukaszewski \& Roney, 2010).

De igual manera, la teoría predice algunos patrones de conducta generales relacionados con la estrategia de emparejamiento perseguida por cada sexo; cada estrategia delimita un conjunto propio de problemas y sus respectivas adaptaciones para enfrentarlos. Por ejemplo, en el emparejamiento a largo plazo, como en el caso de la monogamia, ambos sexos se benefician de la cooperación mutua en la crianza de los hijos y suelen ser un poco más selectivos con sus parejas. Sin embargo, otras formas de emparejamiento a largo plazo son comunes en los humanos; alrededor del $80 \%$ de las sociedades preindustriales presentan sistemas maritales que se asemejan a formas poligínicas de emparejamiento (Ford \& Beach, 1951; Schmitt, 2015). Por otra parte, el emparejamiento a corto plazo en los humanos se manifiesta en conductas relacionadas con la promiscuidad, la infidelidad, la prostitución, las relaciones abiertas y la monogamia serial. De igual manera, como respuesta a regulaciones culturales (i.e., de desaprobación) de las relaciones a corto plazo, esta estrategia puede no ser pública en un número de sociedades (Schmitt, 2014).

La evidencia señala de forma consistente la marcada preferencia por la variedad sexual de los hombres en las relaciones a corto y largo plazo (Buss \& Schmitt, 2019). Asimismo, hay un soporte empírico para las hipótesis que sugieren cómo en el contexto de la reproducción a corto plazo las mujeres prefieren el acceso inmediato a recursos, inician estas relaciones con el fin de evaluar parejas como potenciales relaciones a largo plazo o como forma de búsqueda de mejores parejas a manera de reemplazo de las actuales (Buss et al., 2017).

Hasta el momento se ha hecho referencia indirecta al papel que cumplen las preferencias para la resolución de los problemas reproductivos ancestrales, lo cual tiene sentido si se considera que las preferencias y la selección de parejas están íntimamente relacionadas en el proceso reproductivo. Teóricamente, las preferencias son mecanismos que facilitan la tarea de selección, al brindar un marco sobre qué características de las posibles parejas elegir, además de ofrecer una estimación inicial de la calidad de esos rasgos.

Sin embargo, el problema de la selección no se reduce solo a la atracción; tal como se observa en la figura 1, la integración de las preferencias solo es un paso dentro de un intrincado mecanismo cognitivo que se vale no solo de la información de las potenciales parejas, sino que se nutre y calibra de acuerdo con las condiciones personales, sociales y ecológicas en las que se encuentran inmersos los individuos (Miller \& Todd, 1998). La selección también depende de mecanismos de decisión y heurísticos que pueden modificar la decisión final de emparejarse o acceder a sexo casual; adicionalmente, el proceso esta permeado por fuertes estados emocionales (e.g., deseo sexual, amor) que podrían modular la percepción de la pareja (Förster et al., 2010).

De igual manera, el asunto de la atracción no se limita solo a preferir o rechazar pretendientes, sino que apenas es el primer paso para desencadenar estrategias de persuasión dirigidas a atraer la pareja deseada (i.e., cortejo); asimismo, estar en una relación de pareja implica adoptar estrategias comportamentales que aseguren el mantenimiento de la relación en el caso del largo plazo, e invertir de diversas maneras en los hijos producto de la relación (Daly \& Perry, 2018). Si a eso le sumamos la naturaleza diádica de la reproducción y que la selección es mutua, tenemos que las preferencias podrían estar apenas en la base de la intrincada estructura que representa las relaciones románticas humanas.

Dentro de todo ese complejo panorama de las relaciones de pareja, en el presente texto nos limitamos a ilustrar el papel de las preferencias como mecanismo general para la resolución de problemas reproductivos humanos, ya que, por una parte, estas logran responder, desde la selección misma de parejas, a una gran cantidad de necesidades y exigencias de los sexos (Gangestad \& Simpson, 2000); por otra, la mayoría de investigaciones sobre conducta sexual desde la perspectiva evolutiva se han centrado en el estudio de las preferencias (Conroy-Beam \& Buss, 2016a). No ignoramos el conjunto de problemas reproductivos que ocurren más allá de la atracción y selección inicial de pareja; en cambio, la flexibilidad y el impacto integral de las preferencias sobre aspectos iniciales y futuros de la relación nos permite delimitar el alcance de cuáles retos reproductivos pueden ser solubles desde la vía de la selección, y cuáles requieren adaptaciones específicas para ser abordados.

\section{Preferencias de pareja: una clave para la selección sexual}

Las preferencias de pareja se definen como mecanismos psicológicos especializados que tienen como función 


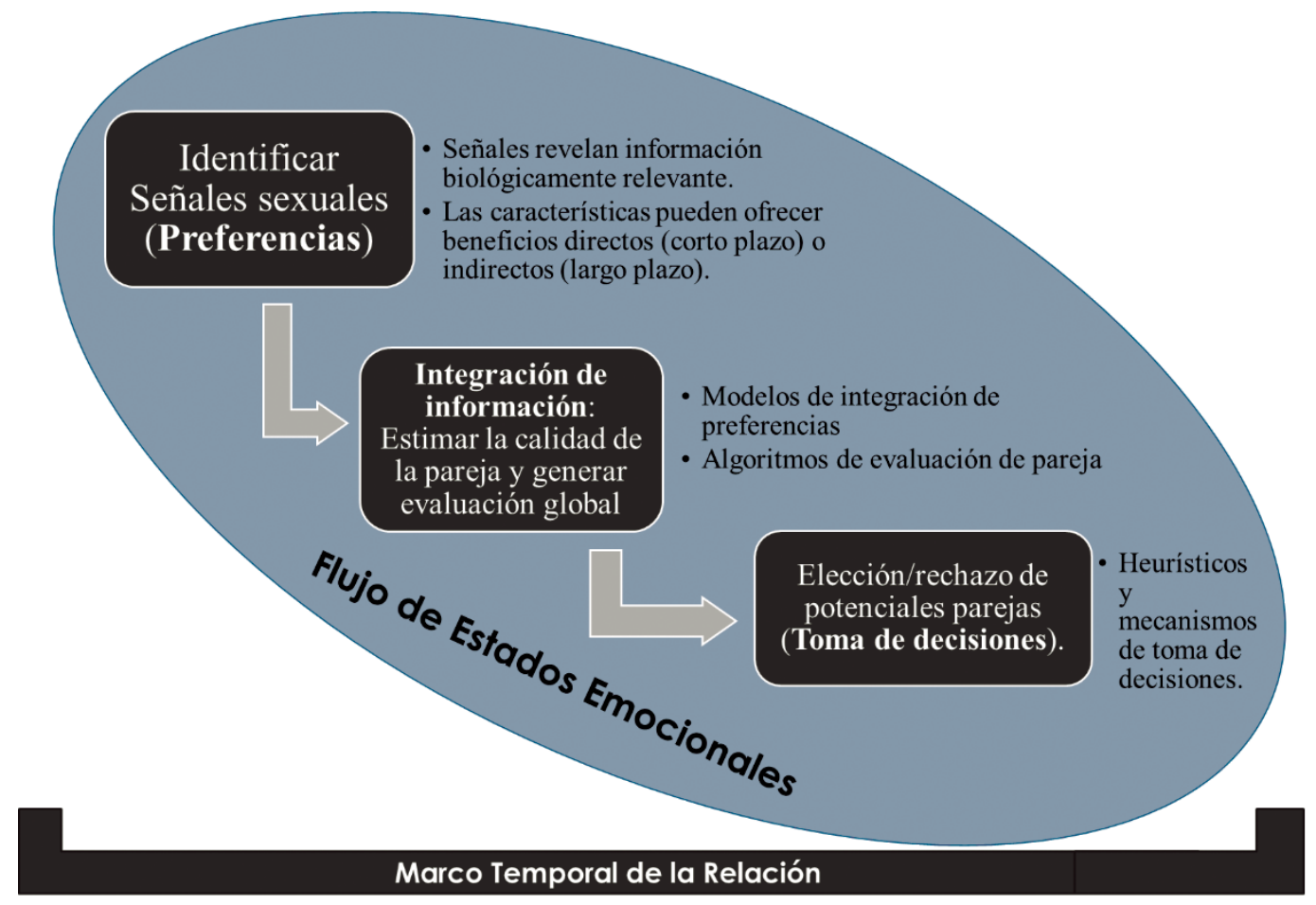

Figura 1. Modelo de procesamiento cognitivo de las preferencias de pareja.

fijar la atención de los individuos en un conjunto particular de características y motivarlos a seleccionar parejas con esas características, con claras consecuencias en la reproducción y un potencial valor adaptativo (Conroy-Beam \& Buss, 2016a).

Las preferencias de pareja son un sistema de evaluación de una gran variedad de rasgos que recaba información biológicamente relevante y se integra en el proceso de selección de pareja, con el fin de guiar la toma de decisiones sobre parejas con características que indiquen una alta eficacia biológica o fitness positivo; este sistema se ha observado en un número de especies animales y humanos (Bateson \& Healy, 2005; Ryan, 2018).

Hombres y mujeres difieren en sus preferencias en la selección de pareja dependiendo de los intereses reproductivos ligados a la inversión parental de cada sexo y la estrategia de emparejamiento (véase la tabla 1). Estas diferencias son de grado más no de tipo; tanto hombres como mujeres sobreenfatizan la predilección por algunos rasgos dependiendo del grado de inversión parental inicial y el contexto sociocultural en el que se encuentran los individuos.

En la tabla 1 se observa que las preferencias asociadas a las relaciones a largo plazo responden a problemas reproductivos enfrentados por los individuos de cada sexo; por ejemplo, las preferencias relacionadas con atractivo físico abordan el problema de la calidad genética, si se considera que aquellas cualidades pueden ser señales de buena salud, vigor y resistencia a enfermedades (Miller, 1998), y que la preferencia por recursos económicos y estatus social por parte de las mujeres ayuda a resolver el problema de la inversión continua de recursos en la relación y la crianza de los hijos, con el fin de equilibrar su inversión previa en la gestación y la lactancia. Es importante aclarar que la tabla reúne dominios generales de preferencia que se manifiestan en señales específicas de atractivo; por ejemplo, el atractivo físico reúne preferencias por rostros, altura, peso,
Tabla 1 Patrones de preferencia en la selección de pareja de acuerdo con los tipos de emparejamiento Tomado de Moreno (2020).

\begin{tabular}{|c|c|c|}
\hline & \multicolumn{2}{|c|}{ Tipo de emparejamiento } \\
\hline & Corto plazo & Largo plazo \\
\hline \multirow{5}{*}{ Hombres } & Variedad en parejas & Atractivo físico \\
\hline & sexuales & Fertilidad \\
\hline & Fertilidad & Valor reproductivo/ \\
\hline & Expresión de interés & juventud \\
\hline & sexual & Compromiso/fidelidad \\
\hline \multirow{6}{*}{ Mujeres } & $\begin{array}{l}\text { Acceso inmediato a } \\
\text { recursos }\end{array}$ & $\begin{array}{l}\text { Habilidad para obtener } \\
\text { recursos }\end{array}$ \\
\hline & Atractivo físico & Amabilidad \\
\hline & Rasgos masculinos & Compromiso/fidelidad \\
\hline & & Habilidades de protección \\
\hline & & Estatus social \\
\hline & & Inteligencia/educación \\
\hline
\end{tabular}

simetría facial y corporal, entre otros. Al final, se evalúa la información integrada de estas señales con el propósito de generar un dictamen de atractivo general sobre el aspecto físico (Bateson \& Healy, 2005; Miller \& Todd, 1998).

La evidencia empírica respalda estos patrones de preferencia a largo plazo. Buss (1989) estudió cerca de 37 culturas alrededor del mundo y encontró que en relaciones a largo plazo las mujeres valoran de forma positiva características asociadas a la adquisición de recursos en hombres, mientras que los rasgos ligados a la capacidad reproductiva fueron mejor valorados por parte de los hombres. Shackelford et al. (2005) hicieron una revisión de la base de datos 
recolectada en la investigación anterior y, mediante técnicas estadísticas de análisis de reducción de factores encontraron cuatro dimensiones universales de las preferencias humanas en relaciones a largo plazo que los autores rotulan como: amor vs. recursos/estatus; confiable/estable vs. atractivo físico/salud; educación/inteligencia vs. compromiso parental; y sociabilidad vs. semejanza de creencias religiosas. De igual manera, encontraron que los hombres dieron mayor prioridad a la categoría atractivo físico/salud, mientras que las mujeres orientaron sus preferencias a la categoría estatus/recursos.

En el caso de las relaciones a corto plazo se advierte que las preferencias favorecen la resolución de retos reproductivos vinculados con la búsqueda de variedad y el número de parejas en los hombres, al preferir mujeres sexualmente accesibles (Gray et al., 2019); la preferencia femenina por hombres con un alto atractivo físico ayuda parcialmente a resolver el problema de compensar la inversión parental inicial de las mujeres en intercambio por recibir genes sobresalientes (Gangestad \& Simpson, 2000).

Hasta este punto, las preferencias parecen ser adaptaciones lo suficientemente versátiles para afrontar varios problemas reproductivos, evitando el costo de implementar estrategias comportamentales como la coerción para acceder a parejas, el engaño, la garantía de compromiso y fidelidad, entre otras (Holden et al., 2014).

Aun así, la sola selección con base en las preferencias puede no ser una estrategia muy fiable si se considera que puede haber cambios a lo largo de la vida de las personas que modifiquen aquellos rasgos que atrajeron en un primer momento a potenciales parejas; por ejemplo, los cambios relacionados con el envejecimiento disminuyen la fertilidad con el paso de los años (Conroy-Beam \& Buss, 2019). Además, mecanismos comportamentales tales como el cortejo o las regulaciones sociales (e.g., los matrimonios arreglados) matizan el papel de las preferencias, ya que la selección posiblemente dependerá tanto de factores externos (e.g., lo socialmente deseable) como de la naturaleza misma de las relaciones, en las cuales dos individuos interactúan persuadiéndose mutuamente para ser seleccionados.

A pesar de lo mencionado, es importante comprender que el valor de las preferencias va más allá de ser una adaptación "económica" y robusta para la solución de varios problemas reproductivos de forma simultánea; también ayudan a indagar sobre información vital de las potenciales parejas, información que es difícil de notar a simple vista a menos que contemos con dispositivos (i. e., adaptaciones) para evaluarla, y que eventualmente tendrá consecuencias tanto en la decisión de emparejarse como en las características heredadas por la descendencia fruto de la relación.

Al mismo tiempo, las preferencias por rasgos específicos pueden ser un fenómeno "accidental", si se considera que algunas características atractivas pueden ser utilizadas con el fin de explotar nuestras capacidades perceptuales (Ryan, 2018), como lo hacen análogamente los vistosos anzuelos para atraer peces. Ambas hipótesis sobre el origen y la función de las preferencias se abordan a continuación.

\section{¿Por qué las preferencias?}

Si aceptamos que las preferencias están orientadas a satisfacer exigencias relacionadas con los distintos grados de inversión parental de cada sexo, resulta importante tener en cuenta que los beneficios o las ventajas que deseamos en una pareja no necesariamente están disponibles a simple vista, sino que más bien nos fijamos en señales indirectas de esos atributos que representan la calidad o aptitud genética.

Por tanto, es importante preguntarse qué ventajas adaptativas pueden ofrecer los “buenos genes". En primer lugar, existe una amplia evidencia de cómo factores genéticos están relacionados con la resistencia a las enfermedades infecciosas o parasitarias (Møller \& Alatalo, 1999; Ridley, 1993); mantener actualizado el material genético por medio de la reproducción con individuos con 'buenos genes' (i.e., genes que contienen las claves de un sistema inmunológico fuerte) resulta una estrategia eficaz para contrarrestar patógenos en la futura descendencia. De igual modo, conectado con las ventajas adaptativas de la reproducción sexual al reducir la carga de mutaciones, tener una marcada preferencia por emparejarse con individuos con una baja carga de mutaciones puede ser una forma de asegurar la calidad genética y la salud de la descendencia (Sefcek et al., 2007).

Entre las estrategias orientadas a evitar individuos con alta carga de mutaciones se incluye mostrar una baja preferencia por sujetos con deformaciones congénitas o infecciosas, además de individuos con parentesco cercano (Al-Shawaf et al., 2018); la endogamia promueve la gestación de niños con enfermedades congénitas, dado que la carga personal de mutaciones se potencia cuando se empareja con individuos con genes muy similares a los propios, lo que también explica por qué en los humanos existe una aparente propensión natural a evitar el incesto y a que este se considere un tabú en la mayoría de sociedades (Ford \& Beach, 1951; Van den Berghe, 1983). Adicionalmente, un cuerpo de estudios relacionados con el complejo mayor de histocompatibilidad (CMH) resaltan el valor de seleccionar parejas con mayor variedad genética, considerando que los hijos podrían heredar sistemas inmunes más fuertes (Roberts et al., 2005).

De acuerdo con las ideas expuestas, podemos preguntarnos cuáles señales son indicadores fidedignos de aptitud genética. Por lo general, los indicadores se manifiestan en una gran diversidad de formas anatómicas o comportamentales que revelan información importante sobre la etapa del desarrollo, la salud, la fertilidad, el estado nutricional, el tamaño, la fuerza, la dominancia, el estatus social, la resistencia a la enfermedad y el vigor.

Si bien no hay un consenso general sobre cuáles rasgos son indicadores fiables de salud, fertilidad y calidad genética en los seres humanos, hay una evidencia creciente de señales de salud ligadas a belleza física (Sugiyama, 2015), atractivo facial basado en su similaridad al promedio de la población, pero no basado en simetría (Rhodes \& Halberstadt, 2002), relación cintura-cadera (mujeres) (Singh, 2002), relación hombros-cintura (hombres) (Braun \& Bryan, 2006), edad (Conroy-Beam \& Buss, 2019), la preferencia por olores corporales en relación con el CMH (Havlíček et al., 2020), y preferencias por la masculinidad o feminidad en rostros $y$ voces (Puts et al., 2012).

En algunas situaciones la relación entre buenos genes y sus rasgos indicadores es indirecta, como lo puede ser el canto de cortejo de las aves o las coloraciones brillantes en sus plumajes; en estos casos, las señales son llamativas perceptualmente y proveen información importante sobre su calidad biológica. Sin embargo, lo anterior genera un problema, ¿cómo confiar en esas señales? Respondiendo a 
esa inquietud, Zahavi y Zahavi (1997) propusieron el principio de hándicap para explicar por qué los rasgos indicadores son difíciles de imitar y se constituyen en señales fidedignas de calidad genética.

El principio de hándicap formula que los rasgos indicadores suelen ser costosos en términos de energía metabólica o merman la capacidad adaptativa de los organismos; no obstante, estas características se convierten en una demostración de alta calidad genética, debido a que solo los individuos sobresalientes pueden asumir estos costos sin afectar sus probabilidades de supervivencia frente a otro competidor. Por ello, los indicadores suelen ser difíciles de falsificar, considerando que los sujetos con baja calidad genética no podrían soportar tales cargas sin afectar su supervivencia.

Las características ostentosas en los humanos parecen seguir los patrones descritos por el principio del hándicap; por ejemplo, en el emparejamiento a corto plazo, algunos comportamientos "extravagantes" como el derroche de recursos económicos o el despliegue virtuoso de alguna habilidad (e.g., artística, deportiva o intelectual) puede ser una señal confiable del potencial personal. De igual modo, las voces graves o los rasgos "marcadamente" masculinos podrían ser indicadores honestos, si se considera que se relacionan con niveles altos de testosterona, la cual, al ser inmunosupresora, podría actuar como hándicap (Arnocky et al., 2018).

Si bien estos atributos pueden no ser indicadores perfectos de calidad genética, todavía pueden contribuir como señales que de forma conjunta con otras características permitan tomar decisiones reproductivas eficientes.

Por otra parte, no todos los indicadores de "belleza física" o atractivo en general siguen la lógica de la demostración de cualidades biológicas sobresalientes. Existe la posibilidad de que los organismos tengan preferencias estéticas por colores y formas poco comunes en sus entornos. Los atributos "estéticamente" atractivos pueden ser preferidos por su heredabilidad en las crías, quienes podrían beneficiarse del atributo (e.g., resistencia inmunológica), y adicionalmente ser preferidos por potenciales parejas futuras (Fuller et al., 2005); esta se denomina la hipótesis del hijo sexy. La atracción se genera en un primer momento por sesgos perceptivos que resaltan ciertos rasgos (Ryan, 2018); además, estos mecanismos pueden facilitar la identificación de señales sexuales que proveen información a los individuos sobre disponibilidad, variabilidad genética y fertilidad.

El sistema de evaluación de preferencias pudo coevolucionar en la forma de adaptaciones perceptuales fijadas genéticamente en cada sexo; adicionalmente, los indicadores estéticos cambian en la medida en que son seleccionados por las parejas, de modo que se vuelven más atractivos en variantes más exageradas o complejas de los rasgos, de acuerdo con los principios de la "selección en cascada". Algunos autores han argumentado que el tamaño del cerebro y la inteligencia humana son el resultado de la "mutua selección" de rasgos comportamentales y preferencias por capacidades intelectuales entre hombres y mujeres (Miller, 2011).

Con respecto a las teorías centradas en rasgos de exhibición estética o aptitud genética, estas pueden ser complementarias en la medida en que los rasgos que se destacan en estos modelos puede coexistir en un mismo individuo o los atributos pueden cumplir varias funciones tanto para indicar calidad genética como para señalar características estéticamente atractivas.
Si bien las ideas mencionadas hacen parte de un cuerpo consolidado de teorías y hallazgos empíricos, es importante precisar que aún persisten problemas notables que suponen un reto teórico para el progreso del área.

\section{Retos contemporáneos en el estudio de las preferencias}

El estudio de las preferencias de parejas bajo la teoría de las estrategias sexuales goza de una gran reputación en el campo de la selección sexual en psicología, biología y áreas relacionadas; sin embargo, en los últimos años ha tenido que responder a retos tanto a nivel explicativo como en las predicciones y limitaciones metodológicas que comprometen los alcances de los principios generales en la teoría. Por una parte, se encuentra el problema de abordar los roles de género y la equidad social y sus efectos en los patrones de preferencia de las sociedades modernas, problema que ha sido tratado por teorías alternas tales como la teoría sociocultural de las preferencias (Zentner \& Eagly, 2015). Por otra, estudios metaanalíticos recientes han llegado a la conclusión de que las preferencias ideales tienen poca capacidad para predecir los rasgos de la pareja definitiva (Eastwick et al., 2014); en otras palabras, existen problemas en el poder predictivo de las preferencias con respecto a las decisiones finales en la selección de pareja.

\section{La cuestión sociocultural}

A partir de los estudios de las preferencias de pareja en el campo de la psicología evolutiva (preferimos este término sobre el de "psicología evolucionista”, cuyo sufijo sugiere un carácter ideológico que claramente no corresponde a esta subdisciplina de la psicología) se han reportado valiosos hallazgos sobre la manera en que los individuos orientan sus estrategias sexuales como resultado de las presiones ecológicas que, se presume, atravesaron los humanos durante su evolución. No obstante, desde otras perspectivas teóricas de corte más sociológico o cultural se argumenta que en ocasiones las teorías evolutivas opacan el impacto que puedan tener las influencias socioculturales en estos procesos; en esos términos, se plantea que las sociedades ofrecen creencias compartidas y regulaciones en torno a las dinámicas de pareja que, a su vez, moldean las preferencias de pareja de cada sexo (Zentner \& Eagly, 2015).

Asumiendo lo anterior, las sociedades contemporáneas presentan una variedad de contextos que se constituyen en nuevas condiciones que modifican nuestras relaciones interpersonales; por ejemplo, durante la Revolución Industrial del siglo XIX los roles de hombres y mujeres en relación con el trabajo y la vida familiar, incluyendo la crianza de los hijos, cambiaron de manera importante y, con esto, las formas y los criterios en la búsqueda de pareja. Posteriormente, durante el siglo XX, cambios laborales y sociales condujeron lentamente a un desplazamiento de los roles de género hacia una mayor equidad de derechos y oportunidades entre hombres y mujeres, lo que produjo un nuevo cambio en las formas de cortejo, selección de pareja y conducta sexual (Tannahill, 1992).

La teoría sociocultural de preferencias de pareja propone que las condiciones de cada cultura moldean las 
preferencias en relación con las restricciones morales y las creencias compartidas que imponen roles específicos en ambos sexos; estos roles pueden ser más o menos equitativos en algunas sociedades, o presentar una gran asimetría en otras. De acuerdo con lo anterior, se pronostica que las preferencias están guiadas por aquellos rasgos "adaptativos" dentro de la cultura que se acomodan a los roles sexuales, es decir, el atractivo estaría mediado por aquellas características valoradas culturalmente como positivas y que sean compatibles con las expectativas culturales que se tienen de cada sexo. En esos términos, las preferencias propuestas por las teorías de corte evolutivo pueden o no ser contingentes con las características analizadas en las teorías socioculturales.

A partir de esos principios es posible argumentar que los individuos buscarían parejas con las que tengan expectativas razonables de vivir cómodamente dentro del nicho cultural y, a su vez, buscarían optimizar los costos y los beneficios al elegir racionalmente parejas con características socialmente apreciadas dentro de la cultura (Zentner \& Eagly, 2015).

Considerando los aspectos que atiende la teoría sociocultural, el problema fundamental de su propuesta se centra en comprender las tendencias de los hombres y las mujeres por preferir parejas que se acomoden a las diferentes restricciones y oportunidades que las sociedades imponen sobre ellos. Se parte de la idea según la cual el ser humano tiene una gran variabilidad conductual y, a su vez, una alta capacidad adaptativa, lo cual le permitió a lo largo de su historia no solo crear numerosas producciones culturales, sino que cimentó la construcción de principios que regulan la conducta de los individuos, incluyendo las dinámicas del emparejamiento humano.

En esta teoría también se sugiere que la división sexual del trabajo cumple un papel importante en la formación de las creencias compartidas relacionadas con los roles de género. Por ejemplo, se asume que en las sociedades preindustriales las mujeres se desenvuelven en actividades domésticas y de crianza, mientras que los hombres trabajan en la obtención de recursos; lo anterior se infiere con base en estereotipos sobre las diferencias físicas de hombres y mujeres, ya que las demandas reproductivas de la inversión parental supedita a las mujeres a las labores de lactancia y crianza, mientras que las capacidades físicas de los hombres les permite realizar esfuerzos físicos constantes para la obtención de recursos (Zentner \& Eagly, 2015).

Otro aspecto que propone la teoría sociocultural menciona cómo el atractivo de las cualidades de proveedor (hombre) y las cualidades domésticas (mujer) reflejan un acuerdo social, debido a que se considera que esos rasgos fueron útiles durante la historia de la humanidad en cada contexto cultural particular; así, a lo largo de las múltiples generaciones de las distintas culturas humanas se generaron los roles de género a partir de sus condiciones contextuales y situaciones históricas (Zentner \& Eagly, 2015).

Lo anterior plantea una predicción de la teoría sociocultural en la cual se menciona que, a mayor equidad de género o mayor semejanza entre los roles de género de las sociedades, más similares serán las preferencias para la selección de parejas en los individuos. Respecto a esa hipótesis, Zentner y Mitura (2012) encontraron que existe una correlación significativa entre los índices de desarrollo relacionados con brechas de género y la magnitud de las diferencias de las preferencias manifiestas por cada sexo. Tal correlación indica que en países en los que existe una mayor brecha económica en los ingresos entre hombres y mujeres, las personas tenderán a mostrar mayores diferencias de grado en cuanto a las preferencias de cada sexo.

Ahora bien, si se asume que las preferencias de pareja tienen un importante componente sociocultural en su determinación, es posible inferir que la variabilidad conductual y la capacidad de adaptación humana conforman las bases para la adopción de estrategias de emparejamiento que sean dependientes del contexto cultural en el que viven los individuos. Las teorías evolutivas difieren en la medida en que se concentran en examinar aquellos patrones de emparejamiento que son estables a lo largo de la historia y son semejantes transculturalmente, con el objetivo de explicar las bases universales del comportamiento sexual.

Desde la perspectiva sociocultural se afirma que el afán de encontrar universales en las tendencias humanas reduce los fenómenos a las hipótesis ligadas a la permanencia de las características ancestrales en los humanos modernos, con la consecuencia de que el campo explicativo solo abarcaría una parte del fenómeno de las preferencias de pareja, de manera que se deja en un segundo plano el componente cultural que pueda permear la consolidación de las preferencias. Alternativamente, la teoría sociocultural ofrece un marco de hipótesis en torno a las variables culturales que pueden modular las preferencias de pareja y no necesariamente riñen con los postulados evolutivos; así, es válido plantear que es necesario tener en cuenta ambos niveles de análisis, a fin de comprender integralmente el fenómeno de las preferencias; en otras palabras, es necesario vincular los distintos niveles de explicación más fundamentales a fin de ofrecer un panorama más completo desde una base evolutiva y sociocultural (Cruz \& Javela, 2018).

Ahora bien, aunque la teoría sociocultural ofrece una perspectiva persuasiva que permite entender las preferencias de pareja, falta un largo camino para examinar sus hipótesis en distintas culturas y grupos sociales; más aún, parece existir evidencia contradictoria respecto a sus principales postulados. Por ejemplo, se ha encontrado que las mujeres tienden a preferir y valorar de forma positiva el estatus y los recursos de su pareja, aun si ellas poseen un estatus alto y viven en condiciones económicas privilegiadas (Buss \& Schmitt, 2019; Zhang et al., 2019).

\section{El poder predictivo de las preferencias}

Recientemente se ha reportado que las preferencias ideales, basadas en las expectativas de una pareja ideal, tienen poca capacidad predictiva con respecto a indicadores como la satisfacción marital, o se encuentra poca relación entre las expectativas ideales y las características de los cónyuges seleccionados. Originalmente se asumía que los individuos que lograban emparejarse con personas que cumplían con sus expectativas o "preferencias ideales" reportaban una alta satisfacción marital, entre otros indicadores positivos de calidad relacional; no obstante, la evidencia acumulada parece no apoyar estas ideas, según indican Eastwick et al. (2014).

Este hecho no solo se remonta al contexto del emparejamiento a largo plazo, sino que también se encuentran 
hallazgos relacionados con los encuentros a corto plazo. Por ejemplo, en un estudio realizado en el contexto de citas rápidas, Todd et al. (2007) encontraron que las preferencias reportadas antes de las citas tenían poco poder predictivo sobre la actual elección de pareja. En el caso de los hombres, el atractivo físico fue un factor que influenció la selección de mujeres, mientras que las mujeres reportaron ser más selectivas en relación con su propio valor de atractivo personal. Este patrón también fue reportado por Eastwick y Finkel (2008), quienes simulando las condiciones de un evento de citas rápidas y evaluando las preferencias antes del evento, encontraron poca relación sobre lo que se esperaba idealmente y la selección final de pareja.

Tratar con estos desafíos a la teoría tradicional ha llevado a los investigadores a ofrecer explicaciones de diversos tipos, desde problemas técnicos con respecto a la metodología para obtener los datos de preferencias (Li \& Meltzer, 2015), hasta la formulación de modelos más robustos o la modificación de modelos existentes para abordar el problema de las predicciones (Conroy-Beam \& Buss, 2016b), lo que ha llevado a que se reexamine la manera como observamos la naturaleza de las preferencias.

Li y Meltzer (2015) señalan algunas posibles razones por las que se encuentra una débil relación entre las preferencias ideales y la elección de pareja final: (1) juicios causales: esta hipótesis sugiere que las personas tienen poca "consciencia" de la manera como escogen parejas, y las preferencias se presentan como criterios plausibles pero poco precisos para explicar por qué les atrae una persona como potencial pareja; (2) la brecha empática: esta idea sugiere que los procesos de atracción ocurren en un estado emocional "caliente", lo que podría afectar la manera como vemos a nuestros pretendientes; esta situación no logra simularse en varios contextos de investigación, ya que en estos contextos las preferencias de pareja pueden ser contempladas en estados emocionales neutros o "fríos", bajo una experiencia más racional.

Por otra parte, revisando más de cerca la naturaleza de la formación de parejas se puede inferir que: (1) la búsqueda de parejas es un proceso dinámico que no se basa solo en lo que deseamos (i.e., preferencias), ya que esto depende de las potenciales parejas disponibles de nuestro círculo social cercano, además de las capacidades para atraer los mejores prospectos disponibles de acuerdo con nuestras preferencias; (2) existe la posibilidad de que los individuos que reúnen todos nuestros requisitos no estén disponibles en el "mercado"; (3) el emparejamiento es inherentemente competitivo, por lo que es posible que nuestra pareja ideal ya esté comprometida en otra relación; (4) la selección es un proceso recíproco que depende de si la pareja deseada también ve atractivo al otro individuo; y (5) existan regulaciones culturales que limiten la selección de parejas a un estándar ideal dentro de esas sociedades (e.g., cánones de belleza).

De igual manera, el análisis de la interacción de las preferencias con la elección final de pareja se puede ver opacada por limitaciones en el diseño metodológico presentadas por los estudios originales: (1) diversidad y representatividad de la muestra: muchos estudios se centran en la población universitaria con promedios de edad cercanos a los 25 años, lo cual puede estar oscureciendo los efectos de las preferencias y cambios de los rasgos en diferentes edades (e.g., fertilidad de mujeres respecto a la edad, acumulación de recursos en hombres respecto a la edad), así como afectar la sensibilidad y validez de los instrumentos; (2) las medidas de preferencias pueden estar contaminadas por percepciones sesgadas de la pareja (e.g., las personas tienden a sobrestimar el atractivo físico de su pareja (Forster et al., 2010); (3) los estudios clásicos suelen utilizar medidas de autorreporte (Schmitt, 2014) que presentan problemas como el sesgo de deseabilidad social (Holtgraves, 2004), o no pueden capturar elementos del constructo de preferencias que están ligados a estados emocionales (los participantes piensan en la atracción como concepto abstracto, a diferencia de la situación real en la que experimentan el deseo).

Ahora bien, lidiar con estos retos requiere una revisión exhaustiva de cómo se están estudiando las preferencias, lo que incluye su conceptualización (i.e., preferencias ideales vs. satisfacción de preferencias), el marco temporal en el que se estudian (i.e., corto o largo plazo), y los métodos usados considerando sus limitaciones y alcances (e.g., autorreporte, experimentos, etnografías, entre otros). Sin embargo, a fin de abordar los problemas de predicción, algunos autores se han enfocado en entender cómo se integran las preferencias dentro de los mecanismos cognitivos para seleccionar pareja.

Se han propuesto modelos de preferencia centrados en el proceso de cómo se integra y evalúa la información a partir de: (1) la sumatoria o acumulación de rasgos que definen el nivel de atractivo (modelo clásico); (2) establecer prioridades en la evaluación de rasgos como necesidades o lujos (modelo de prioridades) (Li et al., 2011); (3) establecer umbrales en los que los rasgos empiezan a ser deseables (modelo aspiracional secuencial) (Miller \& Todd, 1998); o (4) evaluar de manera simultánea las características de la pareja como un todo y establecer un aproximado de qué tipo de características son deseables en relación con las otras (modelo euclidiano). Conroy-Beam y Buss (2016b) decidieron probar los modelos por medio de simulaciones computacionales en un contexto evolutivo, lo cual dio como resultado que el modelo que mejor se adaptó y logró sobrevivir múltiples generaciones fue el modelo de evaluación simultánea o modelo euclidiano de preferencias. Al replicar el modelo con las preferencias de una muestra de participantes en internet y un grupo de recién casados, se encontró un nivel de predicción de satisfacción de preferencias modesto pero positivo en el modelo euclidiano. De igual modo, estos mismos resultados se lograron contrastar con una muestra de participantes residentes en 45 países alrededor del mundo (Conroy-Beam et al., 2019).

Lo anterior puede llevar a pensar que la respuesta a los interrogantes con respecto al poder predictivo de las preferencias puede estar en el estudio de los mecanismos que integran la información de las preferencias para tomar la decisión de optar por una pareja, ya que se dispondría de un método que articula los estándares ideales en relación con el proceso cognitivo de evaluación y elección. Así, encontrar la solución al bajo nivel predictivo de las preferencias con respecto a los indicadores de satisfacción de la pareja actual, parece requerir la conjunción entre la explicación de los mecanismos distales y proximales que aborden las limitaciones de los diseños metodológicos clásicos y considere que las preferencias son apenas el primer paso para comprender el problema de la búsqueda y selección de pareja. 


\section{Conclusiones}

El estudio de la reproducción sexual y sus implicaciones en la conducta de los organismos refleja la historia de un campo con una larga trayectoria de desarrollo y transformaciones, desde que Darwin señaló el valor de la reproducción diferencial como uno de los pilares fundamentales de sus teorías de selección natural y sexual. Si bien esas ideas tuvieron un impacto mixto, o fueron en buena medida ignoradas durante el pasado siglo en las disciplinas sociales (incluyendo la psicología), recientemente existe un renovado interés por la aplicación de principios evolutivos en nuestra conducta sexual y social.

En este momento es posible desarrollar un marco teórico integrativo que permita comprender de manera más comprehensiva el complejo conjunto de fenómenos e implicaciones de la reproducción sexual en el marco de la evolución y la vida social. Para esto, es necesario pensar en los problemas de investigación en términos de causas próximas, del desarrollo, la filogenia y las causas últimas, que permite integrar los mecanismos conductuales y neurobiológicos con una comprensión filogenética y funcional, en este caso, de las preferencias en la selección de pareja.

Los teóricos en el área consideran las preferencias un mecanismo conductual que permite incrementar la eficacia biológica. Si este mecanismo es exclusivamente genético o un producto cultural, no parece ser una formulación adecuada de la pregunta. La literatura de preferencias se ha concentrado bastante en su naturaleza general y transcultural, mostrando un cierto grado de universalidad, lo que sugiere que es un producto evolutivo. A pesar de ello, no resulta razonable o productivo contar con dos explicaciones paralelas del mismo conjunto de fenómenos, por lo que es necesario buscar la integración mencionada, cuando menos, por un acto de honestidad intelectual.

Es fundamental reconocer que un mecanismo motivacional que ha mostrado una fortaleza para influenciar un conjunto importante de fenómenos que excede el ámbito de la selección de pareja no puede comprenderse si no se entiende su valor para mejorar la eficacia biológica.

A partir de esto es posible identificar componentes más o menos flexibles de dicho mecanismo e incluso entender cómo es posible, mediante un proceso de selección de cascada, nuestra preferencia y alta motivación por estímulos con características exageradas, que incluso se conviertan en motores de cambio cultural, con todas sus consecuencias y tensiones.

Si bien las preferencias parecen jugar un papel importante en la selección de pareja, es apresurado exagerar su papel en la capacidad de movilizar la selección última de pareja, o su rol en la satisfacción y eficacia marital. La propia literatura científica realiza desde hace algún tiempo correcciones sobre el entusiasmo inicial acerca de la capacidad predictiva del concepto. Por tanto, es necesario extender la investigación en diversas direcciones que permitan ajustar la teoría de una forma pausada, pero sólida.

Esto demanda reconocer las limitaciones de los datos actuales y extender sus alcances. Así, por ejemplo, estudios sobre el desarrollo de las preferencias a lo largo de la vida son esenciales para comprender no solo influencias contextuales, sino los cambios estratégicos que implica el mecanis- mo de preferencias. De forma similar, algunos mecanismos cognitivos y emocionales fundamentales seguramente juegan un papel que aún no se comprende de forma adecuada en el estudio de las preferencias de parejas, pero que muestran antecedentes en otros contextos de selección. Por ejemplo, las variaciones en las preferencias en contextos de alta complejidad de la oferta de estímulos se viene insinuando y seguramente crecerá en el próximo futuro, así como las variaciones de preferencias en contextos emocionales variados (Moreno, 2020), o el papel modulador de la presencia de otras potenciales parejas en el contexto de elección.

El estudio transcultural de las preferencias debe ir más allá de extender los resultados a otros contextos igualmente jóvenes, urbanos, educados y altamente influenciados por los medios de comunicación occidental. Las dificultades inherentes a los estudios transculturales mantienen limitaciones que no se pueden ignorar a partir de inferencias acerca de la generalidad de la naturaleza humana. El reconocimiento de la riqueza de la experiencia humana no puede ser el arma de una discusión anacrónica entre naturaleza y experiencia. La teoría evolutiva ha llegado a las ciencias del comportamiento con cierta lentitud, pero su capacidad explicativa no resulta fácil de renunciar por razones ideológicas o de otro orden.

Algunos autores han planteado que la selección sexual jugó un papel determinante en la evolución de características humanas distintivas como, por ejemplo, una alta capacidad cognitiva. Esta es una hipótesis valiosa y ubica al mecanismo de preferencias en un papel de privilegio en dicho contexto evolutivo. Otros problemas clásicos de investigación seguramente seguirán siendo de gran interés en el estudio de la selección sexual y, en consecuencia, en el estudio de las preferencias. Quizá ninguno tan relevante como el de las diferencias sexuales, el cual, por razones sociales, se ubica como un problema de alta relevancia y causa de desacuerdo especialmente en las denominadas ciencias sociales.

A pesar de que el estudio de las preferencias aún tiene un largo camino para resolver aspectos conceptuales dentro de la teoría, o refinar los métodos para indagar en los contextos de emparejamiento, los avances en el examen de los mecanismos psicológicos que se integran funcionalmente en el problema de la atracción, además de la consideración de nuevas perspectivas que ofrezcan herramientas de análisis desde la cultura y la sociedad, pueden ser una vía para el desarrollo del campo, en cooperación con las propuestas de otras disciplinas. De igual modo, ahondar en aspectos como el desarrollo de la relación misma, más allá de solo la búsqueda y la atracción de la pareja, parece ser uno de los siguientes pasos para buscar respuestas sobre cómo las preferencias se manifiestan en un contexto en el que, de forma continua, hay un monitoreo de la pareja. El estudio de las preferencias de pareja representa un campo en continuo crecimiento, de modo que es una de las apuestas más prometedoras para entender el mundo de la sexualidad humana.

\section{Agradecimientos}

Los autores agradecen los comentarios y sugerencias de tres revisores anónimos que han contribuido a mejorar este artículo en su alcance, claridad y precisión. 


\section{Conflicto de intereses}

Los autores manifiestan no tener ningún conflicto de intereses.

\section{Referencias}

Al-Shawaf, L., Lewis, D. M., \& Buss, D. M. (2018). Sex differences in disgust: Why are women more easily disgusted than men? Emotion Review, 10(2), 149-160. https://doi.org/10.1177/1754073917709940

Andersson, M. (1994). Sexual selection. Princeton, NJ: Princeton University Press.

Arnocky, S., Hodges-Simeon, C. R., Ouellette, D., \& Albert, G. (2018). Do men with more masculine voices have better immunocompetence? Evolution and Human Behavior, 39(6), 602-610. https://doi.org/10.1016/j.evolhumbehav.2018.06.003

Bateson, M., \& Healy, S. D. (2005). Comparative evaluation and its implications for mate choice. Trends in Ecology \& Evolution, 20(12), 659-664. https://doi.org/10.1016/j.tree.2005.08.013

Bernstein, H., Bernstein, C., \& Michod, R. E. (2012). DNA repair as the primary adaptive function of sex in bacteria and eukaryotes. International Journal of Medical and Biological Frontiers, 18(2/3), 111.

Braun, M. F., \& Bryan, A. (2006). Female waist-to-hip and male waist-to-shoulder ratios as determinants of romantic partner desirability. Journal of Social and Personal Relationships, 23(5), 805-819. https://doi.org/10.1177/0265407506068264

Buss, D. M. (1989). Sex differences in human mate preferences: Evolutionary hypotheses tested in 37 cultures. Behavioral and Brain Sciences, 12, 1-49. https://doi.org/10.1017/S0140525X00023992

Buss, D. M. (2018). Sexual and emotional infidelity: Evolved gender differences in jealousy prove robust and replicable. Perspectives on Psychological Science, 13(2), 155-160. https://doi. org/10.1177/1745691617698225

Buss, D. M., Goetz, C., Duntley, J. D., Asao, K., \& Conroy-Beam, D. (2017). The mate switching hypothesis. Personality and Individual Differences, 104, 143-149. https://doi.org/10.1016/j. paid.2016.07.022

Buss, D. M., \& Shackelford, T. K. (1997). From vigilance to violence: Mate retention tactics in married couples. Journal of Personality and Social Psychology, 72(2), 346-361. https://doi. org/10.1037/0022-3514.72.2.346

Buss, D. M., \& Schmitt, D. P. (1993). Sexual strategies theory: An evolutionary perspective on human mating. Psychological Review, 100(2), 204-232. https://doi.org/10.1037/0033-295X.100.2.204

Buss, D. M., \& Schmitt, D. P. (2019). Mate preferences and their behavioral manifestations. Annual Review of Psychology, 70, 77-110. https://doi.org/10.1146/annurev-psych-010418-103408

Carrillo, S. (2011). Lecciones de amor parental: una perspectiva evolucionista. En G. Gutiérrez \& M. R. Papini (Eds.), Darwin y las ciencias del comportamiento (pp. 393-414). Bogotá: Universidad Nacional de Colombia, Colegio Colombiano de Psicólogos.

Conroy-Beam, D., \& Buss, D. M. (2016a). Mate preferences. En T. K. Shackelford \& V. Weekes-Shackelford (Eds.), Encyclopedia of evolutionary psychological science (pp. 1-11). Suiza: Springer. https://doi.org/10.1007/978-3-319-16999-6_1-1

Conroy-Beam, D., \& Buss, D. M. (2016b). How are mate preferences linked with actual mate selection? Tests of mate preference integration algorithms using computer simulations and actual mating couples. PloS One, 11(6), e0156078. https://doi. org/10.1371/journal.pone.0156078

Conroy-Beam, D., \& Buss, D. M. (2019). Why is age so important in human mating? Evolved age preferences and their influences on multiple mating behaviors. Evolutionary Behavioral Sciences. 13(2), 127-157. https://doi.org/10.1037/ebs0000127

Conroy-Beam, D., Buss, D. M., Asao, K., Sorokowska, A., Sorokowski, P., Aavik, T.,... \& Zupančič, M. (2019). Contrasting computational models of mate preference integration across 45 countries.
Scientific Reports, 9(1), 1-13. https://doi.org/10.1038/s41598019-52748-8

Cruz, J. E., \& Javela, L. G. (2018). Psicología evolucionista, volver a lo natural. En G. Gutiérrez (Ed.), Teorías en psicología. integración y el futuro de la disciplina (pp. 84-98). Bogotá: Manual Moderno, Ascofapsi, Colpsic.

Daly, M., \& Perry, G. (2018). Perspectivas evolucionistas sobre el cuidado y el bienestar infantil. En G. Gutiérrez (Ed.), Teorías en Psicología. Integración y el Futuro de la Disciplina (pp. 134-157). Bogotá: Manual Moderno, Ascofapsi, Colpsic.

Darwin, C. (1871). The Descent of Man, and Selection in Relation to Sex. Londres: John Murray.

Eastwick, P. W., \& Finkel, E. J. (2008). Sex differences in mate preferences revisited: Do people know what they initially desire in a romantic partner? Journal of Personality and Social Psychology, 94(2), 245-264. https://doi.org/10.1037/0022-3514.94.2.245

Eastwick, P. W., Luchies, L. B., Finkel, E. J., \& Hunt, L. L. (2014). The predictive validity of ideal partner preferences: A review and meta-analysis. Psychological Bulletin, 140, 623-665. https://doi.org/10.1037/a0032432

Fisher, R. A. (1930). The genetical theory of natural selection. Oxford: Oxford University Press.

Ford, C. S., \& Beach, F. A. (1951). Patterns of Sexual Behavior. Nueva York: Harper and Row.

Förster, J., Özelsel, A., \& Epstude, K. (2010). How love and lust change people's perception of relationship partners. Journal of Experimental Social Psychology, 46(2), 237-246. https://doi. org/10.1016/j.jesp.2009.08.009

Fuller, R. C., Houle, D., \& Travis, J. (2005). Sensory bias as an explanation for the evolution of mate preferences. The American Naturalist, 166(4), 437-446.

Gangestad, S. W., \& Simpson, J. A. (2000). The evolution of human mating: Trade-offs and strategic pluralism. Behavioral and Brain Sciences, 23, 573-644.

Gray, P. B., Garcia, J. R., \& Gesselman, A. N. (2019). Age-related patterns in sexual behaviors and attitudes among single US Adults: An evolutionary approach. Evolutionary Behavioral Sciences, 13(2), 111-126. https://doi.org/10.1037/ebs0000126

Gutiérrez, G. (2010). Selección sexual, comportamiento y aprendizaje. En E. Zerda (Ed.), Bases biológicas del comportamiento animal y humano (pp. 217-240). Bogotá: Universidad Nacional de Colombia.

Gutiérrez, G., \& Domjan, M. (1996). Learning and male-male sexual competition in Japanese quail (Coturnix japonica). Journal of Comparative Psychology, 110, 170-175. https://doi. org/10.1037/0735-7036.110.2.170

Gutiérrez, G., \& Ortiz, J. (2002). Competencia de esperma en humanos: contexto y mecanismos. Suma Psicológica, 9, 81-106.

Hadany, L., \& Beker, T. (2007). Sexual selection and the evolution of obligatory sex. BMC Evolutionary Biology, 7(1), 245. https:// doi.org/10.1186/1471-2148-7-245

Hadany, L., \& Otto, S. P. (2009). Condition-dependent sex and the rate of adaptation. The American Naturalist, 174(S1), S71-S78.

Hamilton, W. D. (1964). The genetical evolution of social behaviour. II. Journal of Theoretical Biology, 7(1), 17-52.

Havlíček, J., Winternitz, J., \& Roberts, S. C. (2020). Major histocompatibility complex-associated odour preferences and human mate choice: Near and far horizons. Philosophical Transactions of the Royal Society B, 375(1800), 20190260. https://doi. org/10.1098/rstb.2019.0260

Holden, C. J., Zeigler-Hill, V., Pham, M. N., \& Shackelford, T. K. (2014). Personality features and mate retention strategies: Honesty-humility and the willingness to manipulate, deceive, and exploit romantic partners. Personality and Individual Differences, 57, 31-36. https://doi.org/10.1016/j.paid.2013.09.018

Holtgraves, T. (2004). Social desirability and self-reports: Testing models of socially desirable responding. Personality 
and Social Psychology Bulletin, 30(2), 161-172. https://doi. org/10.1177/0146167203259930

Kleiven, O. T., Larsson, P., \& Hobæk, A. (1992). Sexual reproduction in Daphnia magna requires three stimuli. Oikos, 65(2), 197-206. https://doi.org/10.2307/3545010

Li, N. P., \& Meltzer, A. L. (2015). The validity of sex-differentiated mate preferences: Reconciling the seemingly conflicting evidence. Evolutionary Behavioral Sciences, 9(2), 89-106. https:// doi.org/10.1037/ebs0000036

Li, N. P., Valentine, K. A., \& Patel, L. (2011). Mate preferences in the US and Singapore: A cross-cultural test of the mate preference priority model. Personality and Individual Differences, 50(2), 291-294. https://doi.org/10.1016/j.paid.2010.10.005

Lukaszewski, A. W., \& Roney, J. R. (2010). Kind toward whom? Mate preferences for personality traits are target specific. Evolution and Human Behavior, 31(1), 29-38. https://doi.org/10.1016/j. evolhumbehav.2009.06.008

Matthews, R. N., \& Domjan, M. (2011). Selección sexual: competencia de esperma, sistemas de apareamiento y aprendizaje. En G. Gutiérrez \& M. R. Papini (Eds.), Darwin y las ciencias del comportamiento (pp. 115-136). Bogotá: Universidad Nacional de Colombia, Colegio Colombiano de Psicólogos.

Maynard-Smith, J. (1978). The evolution of sex. Cambridge: Cambridge University Press.

Miller, G. F. (1998). A review of sexual selection and human evolution: How mate choice shaped human nature. En C. Crawford \& D. L. Krebs (Eds.), Handbook of Evolutionary Psychology: Ideas, Issues, and Applications (pp. 87-130). Hillsdale, NJ: Lawrence Erlbaum Associates.

Miller, G. F. (2011). The Mating Mind: How Sexual Choice Shaped the Evolution of Human Nature. Nueva York: Anchor Books.

Miller, G. F., \& Todd, P. M. (1998). Mate choice turns cognitive. Trends in Cognitive Sciences, 2(5), 190-198.

Møller, A. P., \& Alatalo, R. V. (1999). Good-genes effects in sexual selection. Proceedings of the Royal Society of London. Series B: Biological Sciences, 266(1414), 85-91. https://doi.org/10.1098/ rspb.1999.0607

Moreno, L. (2020). Estados emocionales y sus efectos en la evaluación de parejas a largo plazo. (Tesis de Maestría). Universidad Nacional de Colombia, Bogotá, Colombia.

Pianka, E. R. (1970). On r-and K-selection. The American Naturalist, 104(940), 592-597.

Puts, D. A., Jones, B. C., \& DeBruine, L. M. (2012). Sexual selection on human faces and voices. Journal of Sex Research, 49(2-3), 227-243. https://doi.org/10.1080/00224499.2012.658924

Rhodes, G., \& Halberstadt, J. (2002). Facial attractiveness: Preferences and possible evolutionary mechanisms. En K. Aoki \& T. Akasawa (Eds.), Human Mate Choice and Prehistoric Premarital Networks (pp. 115-126). Kyoto: International Research Center for Japanese Studies.

Ridley, M. (1993). The Red Queen: Sex and the Evolution of Human Nature. Londres: Penguin.

Roberts, S. C., Little, A. C., Gosling, L. M., Perrett, D. I., Carter, V., Jones, B. C.,... \& Petrie, M. (2005). MHC-heterozygosity and human facial attractiveness. Evolution and Human Behavior, 26(3), 213-226. https://doi.org/10.1016/j.evolhumbehav.2004.09.002

Ryan, M. (2018). A Taste for the Beautiful. The Evolution of Attraction. Princeton, NJ: Princeton University Press.

Schurko, A. M., Neiman, M., \& Logsdon Jr, J. M. (2009). Signs of sex: What we know and how we know it. Trends in Ecology \& Evolution, 24(4), 208-217. https://doi.org/10.1016/j.tree.2008.11.010

Schmitt, D. P. (2014). Evaluating evidence of mate preference adaptations: How do we really know what Homo sapiens sapiens really want? En V. Weekes-Shackelford \& T. Shackelford (Eds.), Evolutionary perspectives on human sexual psychology and behavior (pp. 3-39). Nueva York: Springer. https://doi. org/10.1007/978-1-4939-0314-6_1

Schmitt, D. P. (2015). Fundamentals of human mating strategies. En D. M. Buss (Ed.), The Handbook of Evolutionary Psychology. Hoboken, NJ: John Wiley \& Sons. https://doi. org/10.1002/9781119125563.evpsych111

Sefcek, J. A., Brumbach, B. H., Vasquez, G., \& Miller, G. F. (2007). The evolutionary psychology of human mate choice: How ecology, genes, fertility, and fashion influence mating strategies. Journal of Psychology \& Human Sexuality, 18(2-3), 125-182. https://doi.org/10.1300/J056v18n02_05

Shackelford, T. K., Goetz, A. T., Guta, F. E., \& Schmitt, D. P. (2006). Mate guarding and frequent in-pair copulation in humans. Concurrent or compensatory anti-cuckoldry tactics? Human Nature, 17(3), 239-252. https://doi.org/10.1007/ s12110-006-1007-x

Shackelford, T. K., Schmitt, D. P., \& Buss, D. M. (2005). Universal dimensions of human mate preferences. Personality and Individual Differences, 39(2), 447-458. https://doi.org/10.1016/j. paid.2005.01.023

Simmons, L. W., \& Fitzpatrick, J. L. (2012). Sperm wars and the evolution of male fertility. Reproduction, 144, 519-534. https:// doi.org/10.1530/REP-12-0285

Singh, D. (2002). Waist-to-hip ratio: An indicator of female mate value. En K. Aoki \& T. Akasawa (Eds.), Human Mate Choice and Prehistoric Premarital Networks (pp. 79-99). Kyoto: International Research Center for Japanese Studies.

Sugiyama, L. S. (2015). Physical Attractiveness: An adaptationist perspective. En D. M. Buss (Ed.), The Handbook of Evolutionary Psychology. Nueva York: Wiley. https://doi. org/10.1002/9781119125563.evpsych112

Tannahill, R. (1992). Sex in History. Chelsea, MI: Scarborough House.

Todd, P. M., Penke, L., Fasolo, B., \& Lenton, A. P. (2007). Different cognitive processes underlie human mate choices and mate preferences. Proceedings of the National Academy of Sciences, 104(38), 15011-15016. https://doi.org/10.1073/ pnas.0705290104

Trivers, R. (1972). Parental Investment and Sexual Selection. En B. Campbell (Ed.), Sexual Selection and the Descent of Man 18711971 (pp. 136-179). Chicago: Aldine.

Van den Berghe, P. (1983). Human Inbreeding Avoidance: Culture in Nature. Behavioral and Brain Sciences, 6(1), 91-102. https://doi. org/10.1017/S0140525X00014850

Zahavi, A., \& Zahavi, A. (1997). The Handicap Principle: A Missing Piece of Darwin's Puzzle. Oxford: Oxford University Press.

Zentner, M., \& Eagly, A. H. (2015). A sociocultural framework for understanding partner preferences of women and men: Integration of concepts and evidence. European Review of Social Psychology, 26(1), 328-373. https://doi.org/10.1080/10463283.2 015.1111599

Zentner, M., \& Mitura, K. (2012). Stepping out of the caveman's shadow: Nations' gender gap predicts degree of sex differentiation in mate preferences. Psychological Science, 23, 1176-1185. https://doi.org/10.1177/0956797612441004

Zhang, L., Lee, A. J., DeBruine, L. M., \& Jones, B. C. (2019). Are sex differences in preferences for physical attractiveness and good earning capacity in potential mates smaller in countries with greater gender equality? Evolutionary Psychology, 17(2), 1-6. https://doi.org/10.1177/1474704919852921

Zimmer, C. (2009). On the origin of sexual reproduction. Science, 324(5932), 1254-1256. http://doi.org/10.1126/science.324_1254 